

Open 1 Access
Available online on 15.11.2020 at http://jddtonline.info

\section{Journal of Drug Delivery and Therapeutics}

Open Access to Pharmaceutical and Medical Research

(C) 2011-18, publisher and licensee JDDT, This is an Open Access article which permits unrestricted non-commercial use, provided the original work is properly cited



Review Article

\title{
Multiple Unite Pellet Systems (MUPS) as Drug Delivery model
}

\author{
Saad M. Majeed, Mohammed K. Al-Shaheen, Radhwan N. Al-Zidan* and Sameer M. Mahmood \\ Department of Pharmaceutics, College of Pharmacy, University of Mosul, Iraq
}

\begin{abstract}
Multiparticulate drug delivery systems are mainly oral dosage form, consist of small discrete units that exhibit different characteristics especially in release pattern and drug bioavailability. These systems are represented by granules, pellets, microspheres, microcapsules, and minitablets. Pellets offer high flexibility in the design, formulation, and development of oral dosage forms such as sachet, suspension, capsule, and tablet. Multiparticulate tablets manufactured by compaction of multiple unite pellets is one of the latest and, yet, challenging technologies. Multiparticulate tablets combine the benefits of both a tablet and a pellet-filled capsule in one dosage form but their manufacturing experience many difficulties. The oral multiparticulate products consist of polymer-coated subunits or pellets, which are embedded in an inert excipients' matrix, formulated to overcome the difficulty in administering capsules and improve the physicochemical stability of suspension and offer predictable release and uniform distribution in the gastro-intestinal tract compared to the plain tablet. This review discusses the advantages and drawbacks of MUPS, the properties of an ideal MUPS, various pharmaceutical applications of MUPS, the challenges and key variables that to be considered in the tableting process for successful production of MUPS
\end{abstract}

Keywords: MUPS ${ }^{\circledR}$, Pellets, Multiparticulate tablets.

Article Info: Received 13 Sep 2020; $\quad$ Review Completed 27 Oct 2020; $\quad$ Accepted 06 Nov 2020; $\quad$ Available online 15 Nov 2020

Cite this article as:

Majeed SM, Al-Shaheen MK, Al-Zidan RN, Mahmood SM, Multiple Unite Pellet Systems (MUPS) as Drug Delivery model, Journal of Drug Delivery and Therapeutics. 2020; 10(6):231-235 http://dx.doi.org/10.22270/jddt.v10i6.4389

*Address for Correspondence:

Radhwan N. Al-Zidan, Department of Pharmaceutics, College of Pharmacy, University of Mosul, Iraq

\section{Introduction}

The technology of multiple unite dosage form was initially introduced in the early 1950's. It includes different forms such as pellets, granules, micro-particles (ex. Microspheres and microcapsules), mini-tablets, and mini-depots. These forms play a major role in the design of solid dosage form processes because of their unique properties and flexibility in their production with variable release characteristics. ${ }^{1}$ The term MUPS is an abbreviation for Multiple-Unite Pellets System, however, within the field of the pharmaceutical research and dosage form design the term, in general, refers to MUPS compacted into tablets. Thus, the resulting tablets prepared by compression of polymer-coated pellets are called MUPS dosage form or multiparticulate tablets. The oral route remains the most important one for the administration of pharmaceutical preparations, and tablets of various kinds are the most popular dosage forms available in the market preferred by the physicians and patients. ${ }^{2}$

Pellets can be defined as agglomerates of fine powder consist of a drug and inert excipient that is presented as small, free-flowing, spherical or semi-spherical solid units with range size varying between $0.5-1.5 \mathrm{~mm}$, though other sizes could be produced. Pellets offer controlled-release properties like sustained, delayed also site-specific drug delivery. ${ }^{3}$ Pellet synthesis involves an agglomeration or size enlargement process that helps in the formation of spherical beads or pellets. Pellets are usually coated for the preparation of a modified release dosage form. ${ }^{4}$ The compaction of coated particles into multiparticulate tablets is a difficult task, the principal challenge is maintaining the drug release characteristics after compaction as the polymer coating around the pellets may not withstand the compression force inducing fracture of the polymeric coat or the pellets themselves resulting in drug degradation, irritation of the stomach, or irregular drug release pattern and consequently varying drug bioavailability. 5 Therefore, to protect the coated pellets from such problems, the tableting process required optimizing several key formulation variables such as the nature, and amount of tableting excipients in addition to the type and amount of polymer coating as well as the physical factors of pellets like the size, shape, density, and porosity. ${ }^{6}$ The present review focuses on multiple unit pellets system as a solid dosage form, their techniques formulation and pharmaceutical rational of such multi-particles, as well as focuses on multiparticulate tablets shown its characteristics and 


\section{Majeed et al}

production considering the formulation variables with processing factors. These factors play important role in facilitating the compaction of coated multi-particles into multiparticulate tablets with a limited defect to the polymer coat or damage to the pellets themselves.

\section{Pellets as Solid Dosage Forms}

Generally, multiple unite dosage forms are pharmaceutical preparations that consist of a large number of small discrete units (ex. granules, beads, pellets, microspheres, minitablets, and microcapsules). Each dosage form confers specific beneficial characteristics depending, principally, on formulation processes as they permit flexibility in the formulation and development of the pharmaceutical drug delivery system. Although pellet dosage forms are technically complicated, time-consuming, and costly to
Journal of Drug Delivery \& Therapeutics. 2020; 10(6):216-218 produce, they are widely used because they offer a more sophisticated drug-delivery system with greater advantages over other single unite dosage form counterparts. ${ }^{7}$

Pellets consist of agglomerates of fine powder or granules of bulk drug and excipients formed as a result of pelletization techniques that assist in the formation of spherical beads or pellets which can eventually be coated for preparation of modified-release dosage form. The result of such processes leads to as improvement in flowability, mixing properties, avoiding the generation of excessive dust, and improve the physical and chemical properties of fine powder. ${ }^{8}$ Pellet as a single-unit shows a high degree of flexibility in the design and fabrication of oral dosage forms, as shown in figure (1). Pellets can be packed in sachets, suspended in a liquid, filled in a hard-shell gelatin capsule or disintegrating tablet that quickly release their content of pellets in the stomach.

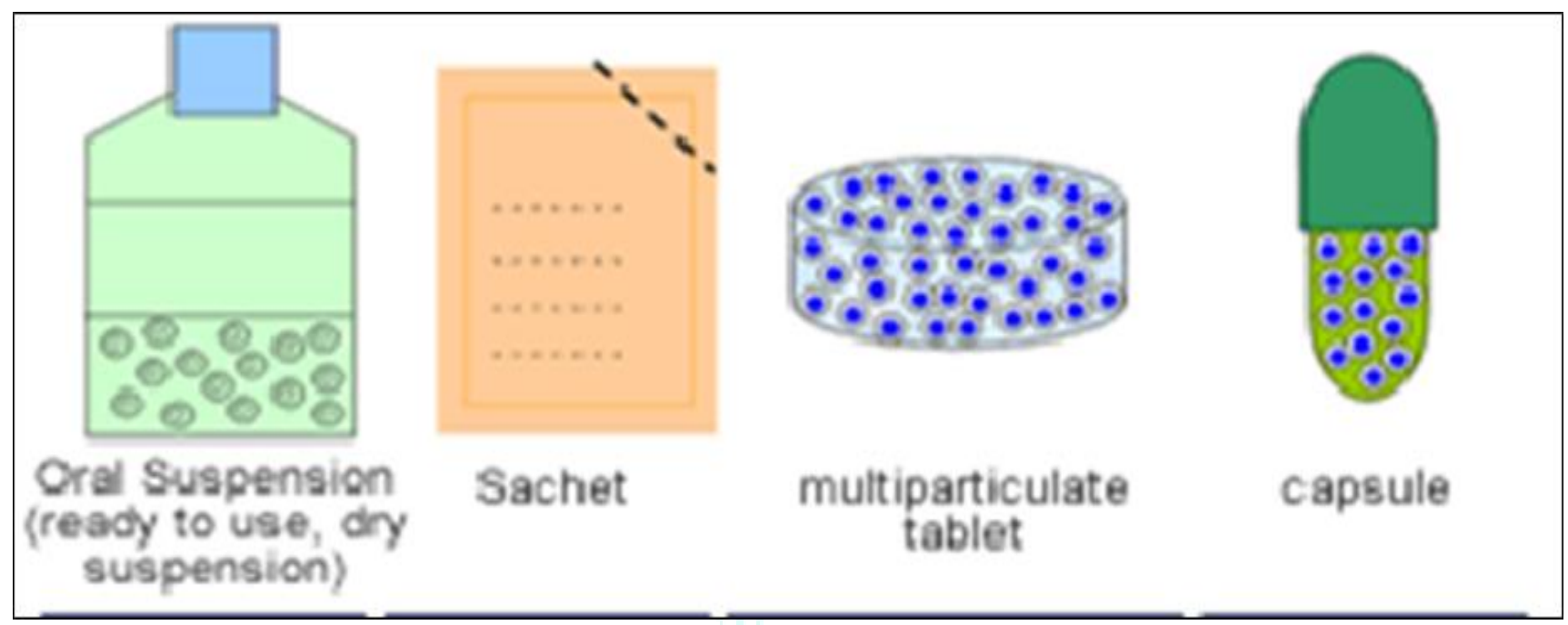

Figure (1): Flexibility of pellets in development of variable dosage forms

Pellets are required for the production of oral controlledrelease dosage forms that have delayed release, sustained release, gastro-resistant, or for targeted drug delivery, to achieve the desired release pattern. Pellets can be modified in multiple ways by using different types of polymers as a coating material, or through the formation of matrix pellets to provide the desired release characteristic in addition to improve the physical properties, chemical stability, and patient acceptance. ${ }^{9}$

\section{Pellets formation techniques}

There are several techniques for the production of pellets in the pharmaceutical industry. The used technique depends on the intended use and the conditions of the manufacturing process. ${ }^{10}$ The different procedures of pellet production are shown in Figure (2).

The extrusion-spheronisation process is the most utilized technique for many reasons. It is a potent, simple, and fast process. Moreover, it allows the incorporation of high amounts of active pharmaceutical ingredients (API). In the extrusion-spheronisation process the pellets are produced from a blend of solids and liquids through a multi-step procedure involving four successive steps:

- Granulation; preparation of the wet mass

- Extrusion; shaping the wet mass into cylinders

- Spheronisation; breaking up the extrudates and rounding of the particles into spheres.

- Desiccation; drying of the pellets

The extrusion step involves forcing the wet mass through an orifice or die beneath a controlled condition. Therefore, forming cylinders or rollers of different sizes called extrudates, then these particles through spheronisation will break into small cylinders and consequently rounded into pellets. ${ }^{11}$ 




A



C

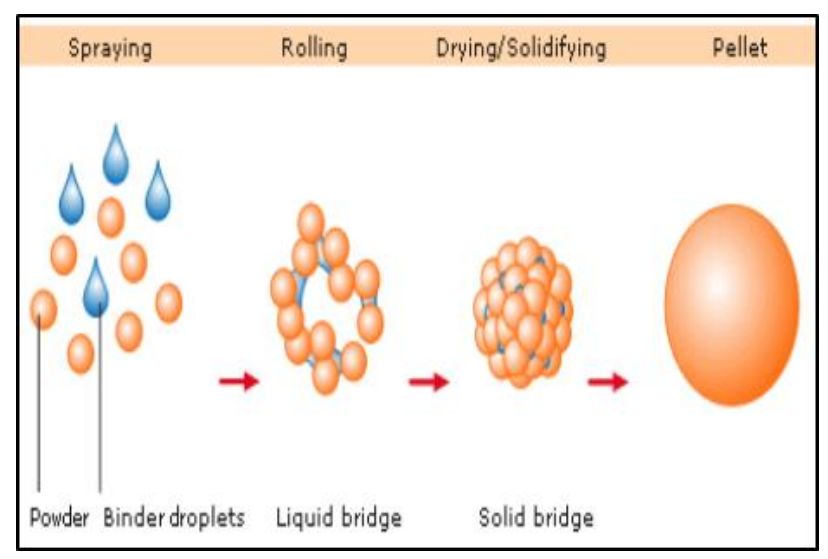

B



D

Figure (2): Different principles of pelletization techniques. (A) Solution/suspension layering, (B) Powder layering (C) Direct pelletization, (D) Extrusion-spheronisation

\section{Ideal Properties of Pellets}

Core pellets are of uniform spherical shape and smooth surface with good flow characteristics. They have a narrow practical size ranged from $0.5 \mathrm{~mm}$ to $1.5 \mathrm{~mm}$, sufficient physical strength, and integrity. Core pellets have an appropriate hardness and low friability that is considered a prerequisite for ease and sufficient coating, prevent segregation during capsule-filling and tablet compression. The high bulk density of pellets is a significant part of achieving content and weight uniformity. For coated pellets with an elegant and uniform thickness of the coating material, and able to achieve the desired drug release characteristics is considered to be perfect pellets. ${ }^{12}$

\section{Pharmaceutical Rational of Pellets}

Pellets can be used as a vehicle for drug delivery with varying release patterns as it can be prepared by many methods, which offer more sophisticated drug delivery systems that provide greater advantages over other singleunit formulations as well as a high degree of flexibility in oral dosage forms design and development. ${ }^{13}$

Therapeutic advantages: oral administration of multiple unites pellet dosage form disperse freely over the gastrointestinal tract (G.I.T) providing a larger surface area for drug absorption. Also, it offers a maximum rate of drug absorption and minimum local irritation to the G.I.T. mucosa due to low drug loading in a single pellet that prevents the risk of dose dumping. Therefore, it improves the safety and efficacy of the medicament and enhances drug bioavailability and patient compliance. ${ }^{14}$
A) Formulation advantages: many oral dosage forms like tablets, capsules, sachet, and suspension can be formulated by using pellets. Principally because it offers greater flexibility in the design and development of API as a pharmaceutical formulation with desired release characteristics providing greater advantages from the same drug formulated as the single-unit dosage form. Such dosage forms can be divided into the desired dose strength without change or adding formulation processing, and can also be blended for simultaneous administration of two or more incompatible bioactive agents with different release profiles within the G.I.T.

Pellets regarded more suitable for manufacture of preparation with acid-sensitive drugs e.g. proton pump inhibitor and masking the taste of unpalatable drugs. ${ }^{15}$

B) Technological advantages: pelletization techniques like layering and extrusion - spheronisation methods offer greater accuracy of uniform drug loading to the pellet. Improve flow property is beneficial in the automated process where exact dosing is required e.g. tableting, capsule filling, and packaging. Low dust formation enhances the process of safety. Pellets of the incompatible drug prepared separately and mixed later or pellets of the same drug but with different release mechanisms can be blended to provide a new modified-release formulation. ${ }^{16}$

\section{Multi-particulate tablet}

Drug delivery system with controlled release characteristics can be classified into single unite dosage form (e.g. matrix 
tablet) and multiple unite dosage forms (e.g. pellets, microsphere) the later dosage forms are technically more complicated, time-consuming, and expensive. Yet they show several advantages over their single unite dosage counterparts. Lately, there has been greater attention in the development of a multi-particulate dosage form as a tablet rather than a hard gelatin capsule or suspension. Compaction of pellets into multiparticulate tablet is one of the more recent and challenging technologies that merge the advantages of both tablets and pellets filled capsules in one dosage form. Multiparticulate dosage forms are consisting of modified release coated subunits embedded in an inert excipient matrix and compressed into a tablet, intended to overcome the difficulties in swallowing the capsule and enhance the physicochemical stability compared to suspension. ${ }^{17}$

The main challenge in the production of multi-particulate tablets is safeguarding the modified drug release characteristic after compaction where the application of the pressure during the compaction process can lead to deformation in the polymer coating or destruction of the pellets themselves and consequently altering the release profile of medicament substances. To protect the coated particulates from such changes, incorporation of inert excipients with cushioning properties is usually used at the tableting process. The extent of changes in the structure of coated particles during compaction depends on many factors including the physical characteristics of the pellets such as the size, shape, porosity, density and also on formulation factors such as the type and amount of polymer coating in addition to the nature and ratio of tableting excipient material, and adjustment of the processing variables especially the compression force and speed of tableting operation. ${ }^{18}$ Considering the previous factors, as well as adjustment of the processing variables especially the compression force and speed of tableting operation will play an essential role in facilitating the compaction of coated multiparticulate into a tablet with little defect or distortion to the coating polymer.



Figure (3): The distribution of the compressed MUPS within the modified-release tablet

\section{Advantages of Multiparticulate Tablet}

The purpose of most recent studies on the compaction of pellets into a tablet is to convert multiple - unite dosage form into a single unite dosage form which is capable to release the primary individual constituents after ingestion. Therefore, combines the benefits of the oral multiple-unit dosage form with those of tablets and show several advantages over pellet-filled capsules or suspended in liquids which involves: 19,20

- Pellets embedded in the inert matrix show greater physicchemical and microbiological stability.

- Lower cost and higher speed of processing in comparison to capsules, using the existing tableting instrument.
- Low dust problem during compression in comparison to conventional tablets.

- Pellets exhibit excellent flow properties due to their spherical shape and thus easy tableting with little segregation problems as compared to conventional granules.

- Easier adjustment of the dose for MUPS since one can readily increase or decrease the dose by adjusting the number of pellets in the formulation.

- Pellets with different release characteristics may be mixed and adjust the drug release profiles for MUPS.

- In comparison to monolithic tablets, dividing the multiparticulate tablet does not affect the release properties since the release characteristics are related to the single subunits.

- The small size of pellets contained in MUPS allows for rapid and uniform transit from the stomach to the small intestine providing better and more drug absorption with greater bioavailability; that results in consistent and controlled pharmacological action with a low tendency for inter-subject variation.

- Sustained release pellets allow a reduction in the dose of drug and dose frequency.

- Several drugs with different release properties can be incorporated into multiple-unit drug delivery systems.

- In comparison to capsules, multiparticulate tablets have the ability to incorporate a large dose of the drug with greater stability.

\section{Ideal Properties of Multiparticulate Tablets}

Ideally, MUPS tablets must possess all the properties of a conventional compressed tablet. Additionally, it should possess the following attributes: ${ }^{21}$

- The drug release from pellets should not be affected by compaction operation.

- The polymer-coated pellets must be able to withstand the pressure during compaction with little deformation.

- The physical strength of the pellet structure must be efficient to withstand the mechanical shocks that are applied in their production, packaging, transport, and dispensing.

- The surface of the compacted MUPS tablets must be smooth and elegant to facilitate the film coating.

- The compacted MUPS should disintegrate rapidly into individual pellets within the G.I.T fluids.

\section{Types of MUPS Formulations}

In general, MUPS formulations can be classified into two categories that illustrated in figure (4):

I- MUPS comprising of coated pellets (figure $4\{A\}$ ); pellets coated with polymer material using different pelletization techniques to achieve the desired release characteristics. These polymer-coated pellets were compacted into multiparticulate tablets either alone or with a mixture of inert excipients.

II- MUPS comprising of matrix pellets (figure $4\{\mathrm{~B}\}$ ); matrix pellets are particles which initially contain excipients that retard drug release by incorporation within the matrix of the pellet structure. This category is less frequently encountered in comparison to the compaction of polymercoated pellets. $^{22}$ 




Figure (4): Type of MUPS: A) MUPS with matrix pellets, B) MUPS with polymer coated pellets

\section{Marketed multiparticulate tablet formulations}

Many drug substances with variable medicinal action have been formulated as MUPS dosage forms, example of such products is shown in table (1):

Table (1): Marketed multiparticulate tablet formulations

\begin{tabular}{|l|l|l|l|l|}
\hline Drug & Product & Company & Therapeutic category & Formulation type \\
\hline Omeprazole magnesium & Losec MUPS & Astra Zeneca & Antiulcer & Delayed release \\
\hline Theophylline & Theodur & Key & Bronchodilator & Extended release \\
\hline Metoprolol tartrate & Toprol XL & Astra Zeneca & Antihypertensive & Extended release \\
\hline Lansoprazole & Prevacid Solu Tab & Takeda & Antiulcer & Delayed release \\
\hline
\end{tabular}

\section{References}

1. Gandhi B, Baheti G. Multiparticulate Drug Delivery Systems; A Review. Inetnational Journal of Pharmaceutical and Chemical Sciences. 2013, 2 (3):1620-1626.

2. Kumar P, Maravaniya J, Deshpande TM, Shinde RV, Katedeshmukh RG. Multiple unite Pellet System - A New Path for drug delivery. International Journal of Universal Pharmacy and Biosciences. 2013; 2(4):401-415.

3. Patel HP, Patel JK, Patel RR, Patel MP. Pellets: A General Overview. International Journal of Pharma World Research. 2010; 1(2).

4. Jagan MK., Venkatesham A., Chandra ME., Vasu K, Kiran Kumar J., Pelletization Techniques for Oral Drug Delivery. International Journal of Pharmaceutical Sciences and Drug Research 2009; 1(2):63-70.

5. Sirisha K, Kvijaya Sri, K Suresh, G. Kamahkar Reddy. Multiple Unite pellet Systems: A review. International Journal of Pharmacy. 2012; 2(2):419-425.

6. V.S.N. Murthery Dwibhashyma, J. Vijaya Ratna. Key formulation variables in tableting of coated pellets. Indian J. Pharm. Sci. 2008; 70(5):555-564.

7. Dukic-Ott A., Thomas De B., Jean Paul R., Willy B., Paul F., Chris V., In-Vitro and In-Vivo Evaluation of Enteric-Coated Starch-Based Pellets Prepared Via Extrusion/Spheronisation.

European Journal of Pharmaceutics and Biopharmaceutics 2008 Volume 70(1):302-312.

8. Mircea Hirjau, Anca Cecilia N., Victoria Hirjau, D. Lupulease, Pelletization Techniques Used in Pharmaceutical Fields. Practica Farmaceutica 2011; 4; 3-4.

9. Hiren P. Patel, J. K. Patel, Ravi R. Patel, Manish P. Patel, PELLETS: A GNERAL OVERVIEW. International Jour nal of Pharma World Research, 2010; 1(2).

10. Kumar V, Mishra SK., Lather AV, Singh R. Multiple Unit Dosage Form-Pellet and Pelletization Techniques: An Overview. International Journal of Research in Ayurveda \& Pharmacy 2011; 2(1):121-125.
11. Sharma A, Chaurasia S. Multiparticulate Drug Delivery System: Pelletization through Extrusion and Spheronization. International Research Journal of Pharmacy 2013; 4(2).

12. Sirisha VR, Vijaya Sri K, Suresh K, Reddy GK, Devanna N. A Review of Pellets and Pelletization Process - A Multiparticulate Drug Delivery System. International Journal of Pharmaceutical Sciences and Research 2013; Vol. 4(6):2145-2158.

13. Gandhi B, Baheti J. Multiparticulates Drug Delivery Systems: A Review. International Journal of Pharmaceutical and Chemical Science, 2013; 2(3).

14. Jawahar N, Patel HA. Multi-Unit Particulates Systems (MUPS): A Novel Pellets for Oral Dosage Forms. Journal of Pharmaceutical Science and Research. 2012; 4(9):1915-1923.

15. Punia S, Bala R, Rana AC. Pelletization Techniques: A Literature Review. Internal Research Journal of Pharmacy 2012; 3(3).

16. Deb R, Ahmed AB. Pellets and Pelletization Techniques: A Critical Review. International Research Journal of Pharmacy 2013; 4(4).

17. Pathik KJ. M., Tanvee MD, Rohit VS., Ramesh GK. Multiple Unit Pellet System - A New Path For Drug Delivery. International Journal of Universal Pharmacy and Bio Sciences 2013; 2(4).

18. Sirisha VR, Vijaya Sri K, Suresh K, Reddy KG. Multiple Unit Pellet Systems: A Review. International Journal of Pharmacy 2012; 2(2): 419-425.

19. Mangesh EB., Shajahan A, Sunil B J., Anil VC., Jayesh MJ., Dinesh MS. MUPS Tablets - A Brief Review. International Journal of Pharm Tech Research 2010; 2(1):847-855.

20. Reddy S, Das P, Ghosh A. MUPS (Multiple Unit Pellet System) Tablets - A Brief Review. Journal of Pharmaceutical and Biomedical Sciences 2011; 12(12).

21. Bhad ME, Abdul S, Jaiswal SB, Chandewar AV, Jain JM, Sakarkar DM. MUPS Tablets - A Brief Review. International Journal of Pharm Tech Research. 2010; 2(1):847-852.

22. Ozarde YS., Serri S, Polshettiwar SA., Kuchekar BS. MultipleUnit-Pellet System (MUPS): A Novel Approach for Drug Delivery. Drug Invention Today, 2012; 4(12):603-609. 